\title{
Editorial for "SON and Automatic Configuration/Optimization in LTE-A Networks: Challenges and Practical Solutions"
}

\author{
Min Zhang ${ }^{1} \cdot$ Michel Kadoch $^{2} \cdot$ Marc St-Hilaire $^{3}$
}

Published online: 1 August 2015

(C) Springer Science+Business Media New York 2015

Editorial:

This special issue features six selected papers with high quality on the topic of SON and Automatic Configuration/ Optimization in LTE-A Networks: Challenges and Practical Solutions. The first article, "A Pricing Power Control Scheme with Statistical Delay QoS Provisioning in Uplink of Two-tier OFDMA Femtocell Networks", co-authored by Shenghua He, Zhaoming Lu, Xiangming Wen, Zhicai Zhang, Jun Zhao and Wenpeng Jing, investigates the interference mitigation problem in LTE-A self-organizing networks (SON). In particular, this work has adopted a price-based power control strategy, where the macrocell base station (MBS) protects itself by pricing the interference from femtocell users (FUs). Based on a mathematical analysis, the authors formulate the problem using game theory and propose an effective power allocation algorithm named PSOPA. The simulation results demonstrate that PSOPA can magnificently improve the average effective capacity of each FU while guaranteeing their statistical delay QoS.

The second article titled "A Stackelberg Game Based Intertier Spectrum Sharing Scheme for LTE-A SON" from Songlin Sun, Liang Gong, Bo Rong, Abdel Mouaki and Amir Basri, develops a spectrum sharing scheme between Micro-eNodeB

Min Zhang

mzhang@bupt.edu.cn

1 State Key Laboratory of IPOC at Beijing University of Posts and Telecommunications (BUPT), Beijing, China

2 Concordia University, Ottawa, Canada

3 Department of Systems and Computer Engineering at Carleton University, Ottawa, Canada
(MeNB) and Pico-eNodeB (PeNB) in LTE-A HetNets. Their proposed model takes into account a number of LTE-A SON techniques, such as eICIC and CRE, and employs Stackelberg game as the mathematical tool. The authors present a variety of simulation results and conclude that their spectrum sharing scheme can allocate frequency resource efficiently between MeNB and PeNB with respect to the change of user density.

The next article investigates spectrum sensing for selforganizing Network. The authors (Mengwei Sun, Shenghong $\mathrm{Li}$, Chenglin Zhao and Bin $\mathrm{Li}$ ) propose a new spectrum sensing scheme to address the grand challenges engendered by time-varying multipath fading channels and unknown noise variance in realistic cognitive radio (CR) applications. CR is of great significance to the future deployment of self-organizing networks due to the spectrum scarcity problem. To make better use of CR, the authors create a dynamic state-space model, based on which spectrum sensing is realized by estimating the primary user's state, multipath channel impulse response and noise variance jointly and iteratively. Numerical results show that the proposed scheme provides a promising solution of spectrum sensing in practical adverse communication environments.

The fourth article, "Data and Control Plane Traffic Modelling for LTE Networks," from Dima Dababneh, Marc St-Hilaire and Christian Makaya, develops a traffic model that involves bandwidth, signalling, voice BHSA (BHVSA), data BHSA (BHDSA), and EPSB. The proposed model provides network operators with realistic traffic parameters at the deployment phase of LTE networks. In particular, those parameters cover different aspects of the traffic and consider a variety of practical factors such as signalling, bandwidth, busy hour session attempts and number of simultaneous EPS (Evolved Packet Systems) bearers. The results of this work serve as an important guidance in the future network planning 
process, and have practical significance in the implementation of LTE SON.

The fifth article, "Physical Layer Security in Cognitive Radio Based Self-Organization Network," from Tianyu Kang and Li Guo, studies the physical layer security problem of a self-organized cognitive radio network (CRN) with a multi-antenna target unknown eavesdropper (TUE). This work endeavors to maximize the worst-user's secrecy rate by designing the collaborative beam former of secondary users. In particular, the authors proved that the power adjustment parameter can be obtained in closed-form, and therefore one can achieve the optimal solution of balancing problem using a two-dimensional search in all cases. Numerical results are presented to justify the significant performance gain from the proposed beamforming strategy over the traditional zeroforcing beamforming.

In the last article entitled "Self-Organizing Scheme Based on NFV and SDN Architecture for Future", the authors (Na Chen, Bo Rong, Abdel Mouaki and Wei Li) highlight the major challenges of future cellular networks and propose to team up network function virtualization (NFV) with software defined networking (SDN) as a solution. In their proposed architecture, NFV centralizes the hardware appliances in the industry standard servers, whereas SDN performs as a core controller of the network. The authors also develop a selforganizing (SO) scheme based on the soft fractional frequency reuse (SFFR) framework to achieve a scalable, energy-saving, and intelligent system.

The guest editors are thankful to our reviewers for their effort in reviewing the manuscripts. We also thank the Editin-Chief, Dr. Imrich Chlamtac for his supportive guidance during the entire process.

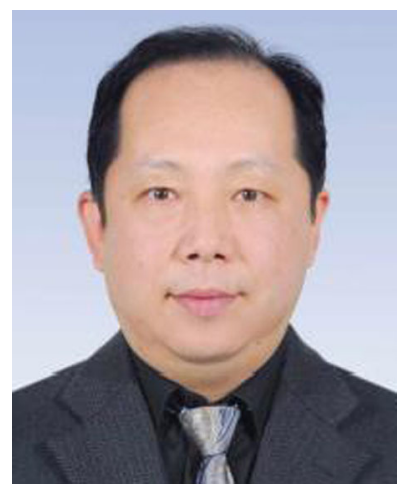

Dr. Min Zhang is currently a Professor in the State Key Laboratory of IPOC at Beijing University of Posts and Telecommunications (BUPT), China, He received his $\mathrm{Ph} . \mathrm{D}$. degree from BUPT in 2003. He has authored or coauthored over 200 technical papers in major international journals and conferences, 12 books in the areas of networks and electromagnetics. He holds 15 China patents. He was elected as the New Century Excellent Talents of Ministry of Education (MOE), China, in 2006, and the Beijing Municipal New Star of Sci\&Tech in 2007 . He received a number of awards for scientific research and technology progress from MOE, China Inst. Comm. (CIC), China Council for Production-Study-Research Cooperation during 2008 2014. He served as Assistant TPC Chair of ACP 2013, Program Committee Member of ChinaCom 2013 and ChinaCom 2014, TPC Co-Chairs of WCSP2014.

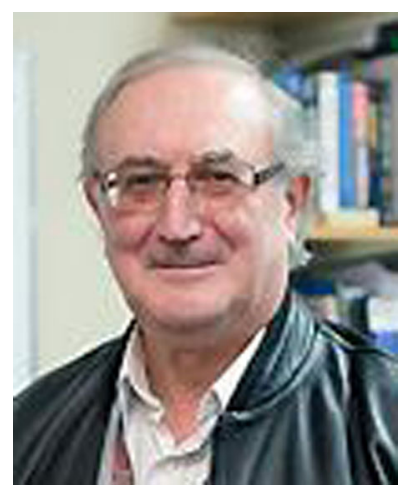

Dr. Michel Kadoch is a full professor at Ecole de technologie superieure ETS (Canada) and the director of the Master Program in engineering. He is active in research mostly in performance analysis and network management and control in wired as well as wireless networks. He is the director of the research laboratory LAGRIT at ETS. He is also an adjunct professor at Concordia University (Canada).He is presently working on Cognitive Radio, Cooperative communication, Cross layer, and Public Safety in wireless Ad hoc and Mesh networks. Professor Kadoch has published many articles and is the author of a book $<$ Protocoles ET reseaux locaux $>$ (Edition ETS, 2012). He is serving as a reviewer for journals and conferences and for grants for NSERC as well as track TPC for ICCCAS, WiMob. He has been involved for many years at ITU-T as a special rapporteur and with the industry namely Teleglobe Canada, CAE, and Communication Canada. He has been a consultant with Harris, Bell South, BC Tel, Concert and British Telecom UK, as well as the CTO (Commonwealth Telecommunication Organization).

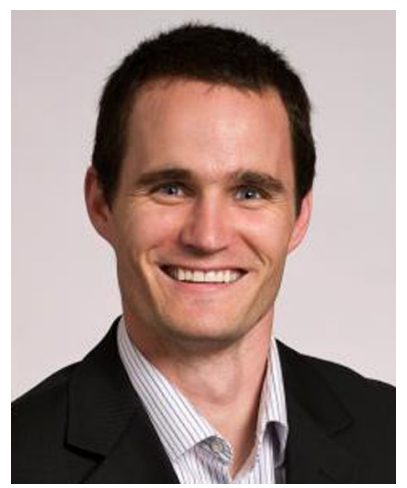

Dr. Marc St-Hilaire is currently an Associate Professor in the School of Information Technology with a cross appointment to the Department of Systems and Computer Engineering at Carleton University, Canada. He is a senior member of the IEEE and is involved in the organisation of several national and international conferences and workshops. His research interests include wireline and wireless networks, mobile computing, performance analysis, telecommunication network planning and mathematical modeling. He authored or co-authored over 90 technical papers. 